\title{
Maximal Regularity for Non-Autonomous Second Order Cauchy Problems
}

\author{
Dominik Dier, El Maati Ouhabaz*
}

April 6, 2018

\begin{abstract}
We consider non-autonomous wave equations

$$
\left\{\begin{array}{l}
\ddot{u}(t)+\mathcal{B}(t) \dot{u}(t)+\mathcal{A}(t) u(t)=f(t) \quad t \text {-a.e. } \\
u(0)=u_{0}, \dot{u}(0)=u_{1} .
\end{array}\right.
$$

where the operators $\mathcal{A}(t)$ and $\mathcal{B}(t)$ are associated with time-dependent sesquilinear forms $\mathfrak{a}(t, .,$.$) and \mathfrak{b}$ defined on a Hilbert space $H$ with the same domain $V$. The initial values satisfy $u_{0} \in V$ and $u_{1} \in H$. We prove well-posedness and maximal regularity for the solution both in the spaces $V^{\prime}$ and $H$. We apply the results to non-autonomous Robin-boundary conditions and also use maximal regularity to solve a quasilinear problem.
\end{abstract}

Key words: Sesquilinear forms, non-autonomous evolution equations, maximal regularity, non-linear heat equations, wave equation.

MSC: 35K90, 35K45, 35K92, 47F05.

\section{Introduction}

The present paper is a continuation of [ADLO13] which is devoted to maximal regularity for first order non-autonomous evolution equations governed by forms. Here we address the problem of maximal regularity for non-autonomous second order problems.

We consider Hilbert spaces $H$ and $V$ such that $V$ is continuously embedded into $H$ and two families of sesquilinear forms

$$
\mathfrak{a}:[0, T] \times V \times V \rightarrow \mathbb{C}, \quad \mathfrak{b}:[0, T] \times V \times V \rightarrow \mathbb{C}
$$

such that $\mathfrak{a}(., u, v):[0, T] \rightarrow \mathbb{C}, \mathfrak{b}(., u, v):[0, T] \rightarrow \mathbb{C}$ are measurable for all $u, v \in V$,

$$
|\mathfrak{a}(t, u, v)| \leq M\|u\|_{V}\|v\|_{V} \quad(t \in[0, T]),
$$

and

$$
\operatorname{Re} \mathfrak{a}(t, u, u)+w\|u\|_{H}^{2} \geq \alpha\|u\|_{V}^{2} \quad(u \in V, t \in[0, T])
$$

\footnotetext{
${ }^{*}$ Corresponding author.
} 
where $M \geq 0, w \in \mathbb{R}$, and $\alpha>0$ are constants. We assume also that $\mathfrak{b}$ satisfies the same properties. For fixed $t \in[0, T]$, we denote by $\mathcal{A}(t), \mathcal{B}(t) \in \mathcal{L}\left(V, V^{\prime}\right)$ the operators associated with the forms $\mathfrak{a}(t, . .$.$) and \mathfrak{b}(t, . .$.$) , respectively. Given a$ function $f$ defined on $[0, T]$ with values either in $H$ or in $V^{\prime}$ and consider the second order evolution equation

$$
\left\{\begin{array}{l}
\ddot{u}(t)+\mathcal{B}(t) \dot{u}(t)+\mathcal{A}(t) u(t)=f(t) \quad t \text {-a.e. } \\
u(0)=u_{0}, \dot{u}(0)=u_{1} .
\end{array}\right.
$$

with initial values $u_{0} \in V$ and $u_{1} \in H$. This is a damped non-autonomous wave equation. The equation without the factor $\dot{u}$, i.e.,

$$
\left\{\begin{array}{l}
\ddot{u}(t)+\mathcal{A}(t) u(t)=f(t) \quad t \text {-a.e. } \\
u(0)=u_{0}, \dot{u}(0)=u_{1} .
\end{array}\right.
$$

is a non-autonomous wave equation.

Our aim is to prove well-posedness and maximal regularity for (1.1) and (1.2). We shall prove three main results. The first one concerns maximal regularity in $V^{\prime}$ for the damped wave equation (1.1). We prove that for $u_{0} \in$ $V, u_{1} \in H$ and $f \in L^{2}\left(0, T, V^{\prime}\right)$ there exists a unique solution $u \in H^{1}(0, T, V) \cap$ $H^{2}\left(0, T, V^{\prime}\right)$. This result was first proved by Lions [Lio61, p. 151] by assuming regularity of $t \mapsto \mathfrak{a}(t, u, v)$ and $t \mapsto \mathfrak{b}(t, u, v)$ for every fixed $u, v \in V$. This regularity assumption was removed in Dautray-Lions [DL88, p. 667], but taking $f \in L^{2}(0, T, H)$ and considering mainly symmetric forms. The general case was given recently by Batty, Chill and Srivastava [BCS08] by reducing the problem to a first order non-autonomous equation. The result in [BCS08] is stated in the case $u_{0}=u_{1}=0$, only. Our proof is different from [BCS08] and is inspired by that of Lions [Lio61]. Next we consider maximal regularity in $H$. This is more delicate and needs extra properties on the forms $\mathfrak{a}$ and $\mathfrak{b}$. We prove that if the forms are symmetric and $t \mapsto \mathfrak{a}(t, u, v)$ and $t \mapsto \mathfrak{b}(t, u, v)$ are piecewise Lipschitz on $[0, T]$ then for $u_{0} \in V, u_{1} \in H$ and $f \in L^{2}(0, T, H)$ there exists a unique solution $u \in H^{1}(0, T, V) \cap H^{2}(0, T, H)$ to the equation (1.1). We also allow some non-symmetric perturbations of $\mathfrak{a}$ and $\mathfrak{b}$. The third result (Theorem 5.1) concerns the wave equation (1.2). We prove that if $\mathfrak{a}$ is symmetric and $t \mapsto \mathfrak{a}(t, u, v)$ is Lipschitz on $[0, T]$, then for every $u_{0} \in V, u_{1} \in H$ and $f \in L^{2}(0, T, H)$ there exists a unique solution $u \in L^{2}(0, T, V) \cap H^{1}(0, T, H) \cap H^{2}\left(0, T, V^{\prime}\right)$ to the equation (1.2). This result is not new and was already proved by Lions [Lio61, p. 150] for the case $u_{0}=0$ and later in [DL88, p. 666] for $u_{0} \in V$ and $u_{1} \in H$. Theorem 5.1 is stated in order to have a complete picture of maximal regularity for wave equations with or without damping. The proof in [DL88] uses a Galerkin method and sectorial approximation. The proofs of the three main theorems use a representation result of Lions (see Theorem 2.4 below) for a given sesquilinear form $E$ acting on a product of a Hilbert and pre-Hilbert spaces $\mathcal{H} \times \mathcal{V}$. In each case we have to define the appropriate spaces $\mathcal{H}, \mathcal{V}$ and the form $E$ to which we apply Theorem 2.4. This idea was already used in [Lio61]. Our choice of the spaces $\mathcal{H}, \mathcal{V}$ and the form $E$ allow us to sharpen and extend some results from [Lio61] and assume less regularity on $t \mapsto \mathfrak{a}(t, u, v)$ and $t \mapsto \mathfrak{b}(t, u, v)$.

We illustrate our abstract results by two examples. The first one is a linear damped wave equation with time dependent Robin boundary conditions. The second is a quasi-linear second order non-autonomous problem. The latter is 
treated by a fixed point argument but the implementation of this classical idea uses heavily a priori estimates that follow from our maximal regularity results for linear equations.

\section{Acknowledgment}

Some ideas in this work germinated during a visit of the second named author at the University of Ulm in the framework of the Graduate School: Mathematical Analysis of Evolution, Information and Complexity financed by the Land BadenWürttemberg and during the visit of the first named author at the University of Bordeaux. Both authors thank Wolfgang Arendt for fruitful discussions on the non-autonomous maximal regularity.

D. Dier is a member of the DFG Graduate School 1100: Modeling, Analysis and Simulation in Economics.

The research of E. M. Ouhabaz is partly supported by the ANR project "Harmonic Analysis at its Boundaries", ANR-12-BS01-0013-02.

\section{Preliminaries}

Throughout this paper, $V$ and $H$ are separable Hilbert spaces over the field $\mathbb{K}=\mathbb{C}$ or $\mathbb{R}$. The scalar products of $H$ and $V$ and the corresponding norms will be denoted by $(. \mid .)_{H},(. \mid .)_{V},\|.\|_{H}$ and $\|.\|_{V}$, respectively. We denote by $V^{\prime}$ the antidual of $V$ when $\mathbb{K}=\mathbb{C}$ and the dual when $\mathbb{K}=\mathbb{R}$. The duality between $V^{\prime}$ and $V$ is denoted by $\langle.,$.$\rangle . Then \langle u, v\rangle=(u \mid v)_{H}$ for $u \in H$ and $v \in V$.

We assume that

$$
V \underset{d}{\hookrightarrow} H
$$

i.e., $V$ is a dense subspace of $H$ such that for some constant $c_{H}>0$,

$$
\|u\|_{H} \leq c_{H}\|u\|_{V} \quad(u \in V) .
$$

By duality and density of $V$ in $H$ one has

$$
H \underset{d}{\hookrightarrow} V^{\prime} .
$$

The space $H$ is then identified with a dense subspace of $V^{\prime}$ (associating to $u \in H$ the antilinear map $v \mapsto(u \mid v)_{H}=\langle u, v\rangle$ for $\left.v \in V\right)$.

Let

$$
\mathfrak{a}:[0, T] \times V \times V \rightarrow \mathbb{K}
$$

be a family of sesquilinear and $V$-bounded forms; i.e.

$$
|\mathfrak{a}(t, u, v)| \leq M\|u\|_{V}\|v\|_{V} \quad(u, v \in V, t \in[0, T])
$$

for some constant $M$, such that $\mathfrak{a}(., u, v)$ is measurable for all $u, v \in V$. We shall call $\mathfrak{a}$ satisfying the above properties a $V$-bounded non-autonomous sesquilinear form. Moreover we say that $\mathfrak{a}$ is quasi-coercive if there exist constants $\alpha>0$, $\omega \in \mathbb{R}$ such that

$$
\operatorname{Re} \mathfrak{a}(t, u, u)+\omega\|u\|_{H}^{2} \geq \alpha\|u\|_{V}^{2} \quad(u \in V, t \in[0, T]) .
$$

If $\omega=0$, we say that the form $\mathfrak{a}$ is coercive. 
For $t \in[0, T]$, a $V$-bounded and quasi-coercive sesquilinear form $\mathfrak{a}(t, . .$.$) is$ closed. The operator $\mathcal{A}(t) \in \mathcal{L}\left(V, V^{\prime}\right)$ associated with $\mathfrak{a}(t, .,$.$) is defined by$

$$
\langle\mathcal{A}(t) u, v\rangle=\mathfrak{a}(t, u, v) \quad \text { for } u, v \in V .
$$

We may also associate with $\mathfrak{a}(t, .,$.$) an operator on H$ by taking the part $A(t)$ of $\mathcal{A}(t)$ on $H$; i.e.,

$$
\begin{aligned}
D(A(t)) & :=\{u \in V: \mathcal{A}(t) u \in H\} \\
A(t) u & :=\mathcal{A}(t) u .
\end{aligned}
$$

Note that if $\mathfrak{a}(t, .,$.$) is symmetric, i.e.,$

$$
\mathfrak{a}(t, u, v)=\overline{\mathfrak{a}(t, v, u)}
$$

for all $u, v \in V$, then the operator $A(t)$ is self-adjoint.

For a Hilbert space $E$ we denote by $L^{2}(0, T, E)$ the $L^{2}$-space on $(0, T)$ of functions with values in $E$ and by $H^{k}(0, T, E)$ we denote the usual Sobolev space of order $k$ of functions on $(0, T)$ with values in $E$. For $u \in H^{1}(0, T ; E)$ we denote the first derivative by $\dot{u}$ and for $u \in H^{2}(0, T ; E)$ the second derivative by $\ddot{u}$.

We start with the following differentiation result.

Lemma 2.1. Let

$$
\mathfrak{a}:[0, T] \times V \times V \rightarrow \mathbb{K}
$$

be a $V$-bounded, quasi-coercive non-autonomous form. Suppose that it is Lipschitz with Lipschitz constant $\dot{M}$, that is

$$
|\mathfrak{a}(t, \phi, \psi)-\mathfrak{a}(s, \phi, \psi)| \leq \dot{M}|t-s|\|\phi\|_{V}\|\psi\|_{V}, t, s \in[0, T] \text { and } \phi, \psi \in V .
$$

Let $u, v \in H^{1}(0, T ; V)$. Then $\mathfrak{a}(., u, v) \in W^{1,1}(0, T)$ and there exists a nonautonomous form $\dot{\mathfrak{a}}$ which is $V$-bounded with constant $\dot{M}$ such that

$$
\mathfrak{a}(., u, v) \cdot \mathfrak{a}(., u, \dot{v})+\mathfrak{a}(., \dot{u}, v)+\dot{\mathfrak{a}}(., u, v)
$$

If additionally $\mathfrak{a}$ is symmetric then

$$
\mathfrak{a}(., u, u)^{\cdot}=2 \operatorname{Re} \mathfrak{a}(., u, \dot{u})+\dot{\mathfrak{a}}(., u, u) .
$$

Note that for $u, v \in V$ we have $\frac{\mathrm{d}}{\mathrm{d} t} \mathfrak{a}(t, u, v)=\dot{\mathfrak{a}}(t, u, v)$ for a.e. $t \in[0, T]$.

This lemma is a consequence of the next two results.

Lemma 2.2. Let $u \in H^{1}(0, T ; V)$ and $v \in H^{1}\left(0, T ; V^{\prime}\right)$. Then $\langle v(),. u().\rangle \in$ $W^{1,1}(0, T)$ and

$$
\langle v(.), u(.)\rangle=\langle\dot{v}(.), u(.)\rangle+\langle v(.), \dot{u}(.)\rangle .
$$

Proof. By Fubini's Theorem we have

$$
\begin{aligned}
\int_{0}^{t}\langle\dot{v}(s), u(s)\rangle \mathrm{d} s & =\int_{0}^{t}\left\langle\dot{v}(s), u(0)+\int_{0}^{s} \dot{u}(r) \mathrm{d} r\right\rangle \mathrm{d} s \\
& =\langle v(t), u(0)\rangle-\langle v(0), u(0)\rangle+\int_{0}^{t} \int_{0}^{s}\langle\dot{v}(s), \dot{u}(r)\rangle \mathrm{d} r \mathrm{~d} s
\end{aligned}
$$




$$
\begin{aligned}
& =\langle v(t), u(0)\rangle-\langle v(0), u(0)\rangle+\int_{0}^{t} \int_{r}^{t}\langle\dot{v}(s), \dot{u}(r)\rangle \mathrm{d} s \mathrm{~d} r \\
& =\langle v(t), u(0)\rangle-\langle v(0), u(0)\rangle+\int_{0}^{t}\langle v(t), \dot{u}(r)\rangle-\langle v(r), \dot{u}(r)\rangle \mathrm{d} r \\
& =\langle v(t), u(t)\rangle-\langle v(0), u(0)\rangle-\int_{0}^{t}\langle v(r), \dot{u}(r)\rangle \mathrm{d} r .
\end{aligned}
$$

Thus

$$
\langle v(t), u(t)\rangle=\langle v(0), u(0)\rangle+\int_{0}^{t}\langle\dot{v}(s), u(s)\rangle \mathrm{d} s+\int_{0}^{t}\langle v(s), \dot{u}(s)\rangle \mathrm{d} s
$$

which proves the claim.

Proposition 2.3. Let $S:[0, T] \rightarrow \mathcal{L}\left(V, V^{\prime}\right)$ be Lipschitz continuous. Then the following assertions hold.

a) There exists a bounded, strongly measurable function $\dot{S}:[0, T] \rightarrow \mathcal{L}\left(V, V^{\prime}\right)$ such that

$$
\frac{\mathrm{d}}{\mathrm{d} t} S(t) u=\dot{S}(t) u \quad(u \in V)
$$

for a.e. $t \in[0, T]$ and

$$
\|\dot{S}(t)\|_{\mathcal{L}\left(V, V^{\prime}\right)} \leq L \quad(t \in[0, T])
$$

where $L$ is the Lipschitz constant of $S$.

b) If $u \in H^{1}(0, T ; V)$, then $S u:=S() u.(.) \in H^{1}\left(0, T ; V^{\prime}\right)$ and

$$
(S u)^{\cdot}=\dot{S}(.) u(.)+S(.) \dot{u}(.) .
$$

Proposition 2.3 is proved in [ADLO13]. Lemma 2.1 follows from (2.4), Lemma 2.2 and Proposition 2.3.

We shall need the following representation result due to Lions. See [Lio59, p. 156], [Lio61, p. 61] or [ADLO13].

Theorem 2.4 (Lions' Representation Theorem). Let $\mathcal{H}$ be a Hilbert space, $\mathcal{V}$ a pre-Hilbert space such that $\mathcal{V} \hookrightarrow \mathcal{H}$. Let $E: \mathcal{H} \times \mathcal{V} \rightarrow \mathbb{K}$ be sesquilinear such that

1) for all $w \in \mathcal{V}, E(., w)$ is a continuous linear functional on $\mathcal{H}$;

2) $|E(w, w)| \geq \alpha\|w\|_{\mathcal{V}}^{2}$ for all $w \in \mathcal{V}$

for some $\alpha>0$. Let $L \in \mathcal{V}^{\prime}$. Then there exists $u \in \mathcal{H}$ such that

$$
L w=E(u, w)
$$

for all $w \in \mathcal{V}$. 


\section{Maximal Regularity for the Damped Wave Equation in $V^{\prime}$}

Let $H, V$ be Hilbert spaces such that $V \stackrel{d}{\hookrightarrow} H$. We define the following maximal regularity space

$$
\begin{aligned}
M R\left(V, V, V^{\prime}\right) & :=L^{2}(0, T, V) \cap H^{1}(0, T ; V) \cap H^{2}\left(0, T ; V^{\prime}\right) \\
& =H^{1}(0, T ; V) \cap H^{2}\left(0, T ; V^{\prime}\right) .
\end{aligned}
$$

Let $\mathfrak{a}:[0, T] \times V \times V \rightarrow \mathbb{C}$ and $\mathfrak{b}:[0, T] \times V \times V \rightarrow \mathbb{C}$ be non-autonomous $V$-bounded and quasi-coercive sesquilinear forms. We denote by $\mathcal{A}(t)$ and $\mathcal{B}(t)$ their associated operators in the sense of (2.4). The following is our first result.

Theorem 3.1. For every $u_{0} \in V, u_{1} \in H$ and $f \in L^{2}\left(0, T ; V^{\prime}\right)$, there exists a unique solution $u \in M R\left(V, V, V^{\prime}\right)$ of the non-autonomous second order Cauchy problem

$$
\left\{\begin{array}{l}
\ddot{u}(t)+\mathcal{B}(t) \dot{u}(t)+\mathcal{A}(t) u(t)=f(t) \quad t \text {-a.e. } \\
u(0)=u_{0}, \dot{u}(0)=u_{1} .
\end{array}\right.
$$

Moreover there exists a constant $C>0$ such that

$$
\|u\|_{M R\left(V, V, V^{\prime}\right)} \leq C\left[\left\|u_{0}\right\|_{V}+\left\|u_{1}\right\|_{H}+\|f\|_{L^{2}\left(0, T ; V^{\prime}\right)}\right] .
$$

As mentioned in the introduction, this theorem was first proved by Lions [Lio61, p. 151] under an additional regularity assumption on $t \mapsto \mathfrak{a}(t, u, v)$ and $t \mapsto \mathfrak{b}(t, u, v)$. This regularity assumption was removed in Dautray-Lions [DL88, p. 667], but taking $f \in L^{2}(0, T, H)$ and considering mainly symmetric forms (they allow some non-symmetric perturbations). Their proof is based on a Galerkin method. Another proof of Theorem 3.1 was given recently by Batty, Chill and Srivastava [BCS08] but they consider only the case $u_{0}=u_{1}=0$. Our proof is based on Theorem 2.4 and is in the spirit of Lions [Lio61]. It is different from the proofs in [DL88] and [BCS08].

A classical result of Lions says that

$$
M R\left(V, V^{\prime}\right):=L^{2}(0, T, V) \cap H^{1}\left(0, T ; V^{\prime}\right) \hookrightarrow C([0, T] ; H),
$$

and also that for $u \in M R\left(V, V^{\prime}\right)$ the function $\|u(.)\|_{H}^{2}$ is in $W^{1,1}(0, T)$ with

$$
\left(\|u\|_{H}^{2}\right)^{\cdot}=2 \operatorname{Re}\langle\dot{u}, u\rangle
$$

see [Sho97, p. 106] and [DL88, p.570]. This implies that $M R\left(V, V, V^{\prime}\right) \hookrightarrow$ $C([0, T] ; V) \cap C^{1}([0, T] ; H)$. Thus for $u \in M R\left(V, V, V^{\prime}\right)$, both $u(0)$ and $\dot{u}(0)$ make sense.

We start with the following basic lemma.

Lemma 3.2. For $v \in H^{1}(0, T ; V)$ we have

$$
\left(\int_{0}^{T}\|v(t)\|_{V}^{2} \mathrm{~d} t\right)^{1 / 2} \leq T\left(\int_{0}^{T}\|\dot{v}(s)\|_{V}^{2} \mathrm{~d} s\right)^{1 / 2}+\sqrt{T}\|v(0)\|_{V} .
$$


Proof. Note that $v(t)=v(0)+\int_{0}^{t} \dot{v}(s) \mathrm{d} s$, thus

$$
\begin{aligned}
\int_{0}^{T}\|v(t)\|_{V}^{2} \mathrm{~d} t & =\int_{0}^{T}\left(v(0)+\int_{0}^{t} \dot{v}(s) \mathrm{d} s \mid v(t)\right)_{V} \mathrm{~d} t \\
& =\int_{0}^{T} \int_{s}^{T}(\dot{v}(s) \mid v(t))_{V} \mathrm{~d} t \mathrm{~d} s+\int_{0}^{T}(v(0) \mid v(t))_{V} \mathrm{~d} t \\
& \leq \int_{0}^{T} \int_{0}^{T}\|\dot{v}(s)\|_{V}\|v(t)\|_{V} \mathrm{~d} t \mathrm{~d} s+\int_{0}^{T}\|v(0)\|_{V}\|v(t)\|_{V} \mathrm{~d} t \\
& \leq \int_{0}^{T}\|v(t)\|_{V} \mathrm{~d} t\left(\int_{0}^{T}\|\dot{v}(s)\|_{V} \mathrm{~d} s+\|v(0)\|_{V}\right) \\
& \leq\left(T \int_{0}^{T}\|v(t)\|_{V}^{2} \mathrm{~d} t\right)^{1 / 2}\left(\left(T \int_{0}^{T}\|\dot{v}(s)\|_{V}^{2} \mathrm{~d} s\right)^{1 / 2}+\|v(0)\|_{V}\right) .
\end{aligned}
$$

Proof of Theorem 3.1. It suffices to show that there exists a unique solution in the case where $T<T_{0}$ and $T_{0}>0$ is a constant that depends only on the constants $M, \omega$ and $\alpha$ of (2.2) and (2.3). Indeed we can extend this solution to $[0, T]$ for any fixed $T$ as follows. We write the interval $[0, T]$ as a finite union of sub-intervals $\left[\tau_{i}, \tau_{i+1}\right]$, each has length less than $T_{0}$. On each interval $\left[\tau_{i}, \tau_{i+1}\right]$ we have a unique solution $u^{i}$ with $u^{i}\left(\tau_{i}\right) \in V, \dot{u}^{i}\left(\tau_{i}\right) \in H$ and $u^{i} \in$ $M R\left(V, V, V^{\prime}\right) \hookrightarrow C^{1}\left(\left[\tau_{i}, \tau_{i+1}\right] ; H\right) \cap C\left(\left[\tau_{i}, \tau_{i+1}\right] ; V\right)$. On $\left[\tau_{i+1}, \tau_{i+2}\right]$ we solve the equation with $u^{i+1}\left(\tau_{i+1}\right)=u^{i}\left(\tau_{i+1}\right)$ and $\dot{u}^{i+1}\left(\tau_{i+1}\right)=\dot{u}^{i}\left(\tau_{i+1}\right)$. We define $u$ on $[0, T]$ by $u=u^{i}$ on $\left[\tau_{i}, \tau_{i+1}\right]$ and check easily that $u \in M R\left(V, V, V^{\prime}\right)$ (on $[0, T]$ ) is the unique solution to (3.1).

We prove existence of a solution in the case where

$$
T<T_{0}=\min \left\{\frac{\alpha^{2}}{M^{2}}, \frac{\alpha}{\sqrt{2} M}\right\} .
$$

Note that we may assume throughout this proof that the forms $\mathfrak{a}$ and $\mathfrak{b}$ are both coercive. Indeed, set $v(t)=e^{w t} u(t)$ then we have

$$
\begin{aligned}
& \ddot{v}(t)+\mathcal{B}(t) \dot{v}(t)+\mathcal{A}(t) v(t) \\
&=e^{w t}\left[\ddot{u}(t)+(\mathcal{B}(t)+2 w) \dot{u}(t)+\left(\mathcal{A}(t)+w \mathcal{B}(t)+w^{2}\right) u(t)\right] .
\end{aligned}
$$

Since $\mathfrak{a}$ and $\mathfrak{b}$ are quasi-coercive, we may choose $w$ large enough such that $\mathfrak{b}+2 w$ and $\mathfrak{a}+w \mathfrak{b}+w^{2}$ are coercive. Note also that $v \in M R\left(V, V, V^{\prime}\right)$ if and only if $u \in M R\left(V, V, V^{\prime}\right)$.

We define the Hilbert space $\mathcal{H}:=H^{1}(0, T ; V)$ endowed with its usual norm $\|u\|_{\mathcal{H}}:=\|u\|_{H^{1}(0, T ; V)}$ and the pre-Hilbert space

$$
\mathcal{V}:=\left\{v \in H^{2}(0, T ; V): \dot{v}(T)=0\right\}
$$

with norm $\|\cdot\|_{\mathcal{V}}:=\|\cdot\|_{\mathcal{H}}$. Further we define the sesquilinear form $E: \mathcal{H} \times \mathcal{V} \rightarrow \mathbb{C}$ by

$$
\begin{aligned}
E(u, v):= & -\int_{0}^{T}(\dot{u} \mid \ddot{v})_{H} \mathrm{~d} t+\int_{0}^{T} \mathfrak{b}(t, \dot{u}, \dot{v}) \mathrm{d} t \\
& +\int_{0}^{T} \mathfrak{a}(t, u, \dot{v}) \mathrm{d} t+\mathfrak{a}(0, u(0), v(0))
\end{aligned}
$$


and for $u_{0} \in V, u_{1} \in H$ and $f \in L^{2}\left(0, T ; V^{\prime}\right)$ we define $F: \mathcal{V} \rightarrow \mathbb{C}$ by

$$
F(v):=\int_{0}^{T}\langle f, \dot{v}\rangle \mathrm{d} t+\mathfrak{a}\left(0, u_{0}, v(0)\right)+\left(u_{1} \mid \dot{v}(0)\right)_{H} .
$$

We claim that

1) $E(., v) \in \mathcal{H}^{\prime}$ and $F \in \mathcal{V}^{\prime}$;

2) $E$ is coercive; i.e., there exists a $C>0$ such that $|E(v, v)| \geq C\|v\|_{\mathcal{H}}^{2}$ for all $v \in \mathcal{V}$.

Suppose for a moment that 1) and 2) are satisfied. Then we can apply Lions's representation theorem (see Theorem 2.4) and obtain $u \in \mathcal{H}$ such that

$$
E(u, v)=F(v) \quad \forall v \in \mathcal{V} .
$$

We show that $u$ is a solution of (3.1).

Let $\psi(t) \in \mathcal{D}(0, T)$ and $w \in V$ and choose $v(t):=\int_{0}^{t} \psi(s) \mathrm{d} s w$. It follows from (3.7) that

$$
-\int_{0}^{T}\langle\dot{u}(t), w\rangle \dot{\psi}(t) d t=\int_{0}^{T}\langle f(t)-\mathcal{B}(t) \dot{u}-\mathcal{A}(t) u(t), w\rangle \psi(t) \mathrm{d} t .
$$

This means that $\dot{u} \in H^{1}\left(0, T ; V^{\prime}\right)$, hence $u \in M R\left(V, V, V^{\prime}\right)$ and

$$
\ddot{u}(t)+\mathcal{B}(t) \dot{u}(t)+\mathcal{A}(t) u(t)=f(t) \quad t \text {-a.e. }
$$

in $V^{\prime}$. For general $v \in \mathcal{V}$, we use again (3.7) and integration by parts to obtain

$$
\begin{aligned}
(\dot{u}(0) \mid & \dot{v}(0))_{H}+\int_{0}^{T}\langle\ddot{u}, \dot{v}\rangle \mathrm{d} t+\int_{0}^{T} \mathfrak{b}(t, \dot{u}, \dot{v}) \mathrm{d} t+\int_{0}^{T} \mathfrak{a}(t, u, \dot{v}) \mathrm{d} t \\
& +\mathfrak{a}(0, u(0), v(0))=\int_{0}^{T}\langle f, \dot{v}\rangle \mathrm{d} t+\mathfrak{a}\left(0, u_{0}, v(0)\right)+\left(u_{1} \mid \dot{v}(0)\right)_{H} .
\end{aligned}
$$

This equality together with (3.8) imply that

$$
(\dot{u}(0) \mid \dot{v}(0))_{H}+\mathfrak{a}(0, u(0), v(0))=\mathfrak{a}\left(0, u_{0}, v(0)\right)+\left(u_{1} \mid \dot{v}(0)\right)_{H} .
$$

Since $v \in \mathcal{V}$ is arbitrary we obtain that $u(0)=u_{0}$ and $\dot{u}(0)=u_{1}$. Therefore, $u$ is a solution of (3.1) on $[0, T]$ for $T \leq T_{0}$ and $T_{0}$ is such that the above properties 1) and 2) are satisfied.

Now we return to 1 ) and 2). Property 1 ) is obvious. We show the coercivity property 2). Let $v \in \mathcal{V}$. The equality $\frac{\mathrm{d}}{\mathrm{d} t}\|\dot{v}(t)\|_{H}^{2}=2 \operatorname{Re}(\ddot{v}(t) \mid \dot{v}(t))_{H}$ implies

$$
\int_{0}^{T} \operatorname{Re}(\ddot{v} \mid \dot{v})_{H} \mathrm{~d} t=-\frac{1}{2}\|\dot{v}(0)\|_{H}^{2} .
$$

It follows that

$$
\begin{aligned}
|E(v, v)| & \geq \operatorname{Re} E(v, v) \\
& =\frac{1}{2}\|\dot{v}(0)\|_{H}^{2}+\int_{0}^{T} \operatorname{Re} \mathfrak{b}(t, \dot{v}, \dot{v}) \mathrm{d} t
\end{aligned}
$$




$$
+\int_{0}^{T} \operatorname{Re} \mathfrak{a}(t, v, \dot{v}) \mathrm{d} t+\operatorname{Re} \mathfrak{a}(0, v(0), v(0)) .
$$

We use coercivity of $\mathfrak{b}, \mathfrak{a}$ and $V$-boundedness of $\mathfrak{a}$ to obtain

$$
\begin{aligned}
|E(v, v)| \geq & \frac{1}{2}\|\dot{v}(0)\|_{H}^{2}+\alpha \int_{0}^{T}\|\dot{v}\|_{V}^{2} \mathrm{~d} t \\
& -M \int_{0}^{T}\|v\|_{V}\|\dot{v}\|_{V} \mathrm{~d} t+\alpha\|v(0)\|_{V}^{2} .
\end{aligned}
$$

Therefore, by Young's inequality, we have

$$
|E(v, v)| \geq \frac{1}{2}\|\dot{v}(0)\|_{H}^{2}+\frac{\alpha}{2} \int_{0}^{T}\|\dot{v}\|_{V}^{2} \mathrm{~d} t-\frac{M^{2}}{2 \alpha} \int_{0}^{T}\|v\|_{V}^{2} \mathrm{~d} t+\alpha\|v(0)\|_{V}^{2} .
$$

Next we apply Lemma 3.2 to obtain

$$
|E(v, v)| \geq\left(\frac{\alpha}{2}-\frac{M^{2} T^{2}}{\alpha}\right) \int_{0}^{T}\|\dot{v}\|_{V}^{2} \mathrm{~d} t+\left(\alpha-\frac{M^{2} T}{\alpha}\right)\|v(0)\|_{V}^{2} .
$$

Now we use (3.5) and the fact that by Lemma $3.2, \int_{0}^{T}\|v\|_{V}^{2} \mathrm{~d} t$ is dominated (up to a constant) by $\int_{0}^{T}\|\dot{v}\|_{V}^{2} \mathrm{~d} t+\|v(0)\|_{V}^{2}$. We obtain 2).

Next we prove uniqueness. Suppose that $u$ and $v$ are two solutions of (3.1) which are in $M R\left(V, V, V^{\prime}\right)$. Set $w=u-v$. Clearly $w \in M R\left(V, V, V^{\prime}\right)$ and satisfies (in $V^{\prime}$ )

$$
\ddot{w}(t)+\mathcal{B}(t) \dot{w}(t)+\mathcal{A}(t) w(t)=0, \quad w(0)=0, \dot{w}(0)=0 .
$$

We show that $w=0$. For fixed $t \in(0, T]$ we have

$$
\int_{0}^{t} \operatorname{Re}\langle\ddot{w}, \dot{w}\rangle \mathrm{d} s+\int_{0}^{t} \operatorname{Re} \mathfrak{b}(s, \dot{w}, \dot{w}) \mathrm{d} s+\int_{0}^{t} \operatorname{Re} \mathfrak{a}(s, w, \dot{w}) \mathrm{d} s=0 .
$$

Using (3.4) we have

$$
\int_{0}^{t} \operatorname{Re}\langle\ddot{w}, \dot{w}\rangle \mathrm{d} s=\frac{1}{2} \int_{0}^{t}\left(\|\dot{w}\|_{H}^{2}\right)^{\cdot} \mathrm{d} s=\frac{1}{2}\|\dot{w}(t)\|_{H}^{2}-\frac{1}{2}\|\dot{w}(0)\|_{H}^{2}=\frac{1}{2}\|\dot{w}(t)\|_{H}^{2},
$$

and hence

$$
\begin{aligned}
0 & =\frac{1}{2}\|\dot{w}(t)\|_{H}^{2}+\int_{0}^{t} \operatorname{Re} \mathfrak{b}(s, \dot{w}, \dot{w}) \mathrm{d} s+\int_{0}^{t} \operatorname{Re} \mathfrak{a}(s, w, \dot{w}) \mathrm{d} s \\
& \geq \frac{1}{2}\|\dot{w}(t)\|_{H}^{2}+\alpha \int_{0}^{t}\|\dot{w}\|_{V}^{2} \mathrm{~d} s-M \int_{0}^{t}\|w\|_{V}\|\dot{w}\|_{V} \mathrm{~d} s .
\end{aligned}
$$

Here we used coercivity of $\mathfrak{b}$ and $V$-boundedness of $\mathfrak{a}$. Therefore, by Lemma 3.2, we have

$$
\begin{aligned}
0 & \geq \frac{1}{2}\|\dot{w}(t)\|_{H}^{2}+\alpha \int_{0}^{t}\|\dot{w}\|_{V}^{2} \mathrm{~d} s-M\left(\int_{0}^{t}\|w\|_{V}^{2} \mathrm{~d} s\right)^{1 / 2}\left(\int_{0}^{t}\|\dot{w}\|_{V}^{2} \mathrm{~d} s\right)^{1 / 2} \\
& \geq \frac{1}{2}\|\dot{w}(t)\|_{H}^{2}+(\alpha-M T) \int_{0}^{t}\|\dot{w}\|_{V}^{2} \mathrm{~d} s
\end{aligned}
$$


By (3.5) we obtain that $w=0$. This shows uniqueness.

Finally, in order to prove the apriori estimate (3.2), we consider the operator

$$
S: V \times H \times L^{2}\left(0, T, V^{\prime}\right) \mapsto M R\left(V, V, V^{\prime}\right), \quad\left(u_{0}, u_{1}, f\right) \mapsto u .
$$

This is a linear operator which is well defined thanks to the uniqueness of the solution $u$ of (3.1). It is easy to see that $S$ is a closed operator. Therefore it is continuous by the closed graph theorem. This gives (3.2).

The previous proof does not give any information on the constant $C$ in (3.2). For small time $T$ one can prove that $C$ depends only on the constants of the forms. This observation will be needed in our application to a quasi-linear problem.

Proposition 3.3. If $T>0$ is small enough, then the constant $C$ in (3.2) depends only on the constants $w, \alpha, M$ and $T$.

Proof. Let $u \in M R\left(V, V, V^{\prime}\right)$ be the solution of (3.1). For fixed $t \in(0, T]$ we have

$$
\int_{0}^{t} \operatorname{Re}\langle f, \dot{u}\rangle \mathrm{d} s=\int_{0}^{t} \operatorname{Re}\langle\ddot{u}, \dot{u}\rangle \mathrm{d} s+\int_{0}^{t} \operatorname{Re} \mathfrak{b}(s, \dot{u}, \dot{u}) \mathrm{d} s+\int_{0}^{t} \operatorname{Re} \mathfrak{a}(s, u, \dot{u}) \mathrm{d} s .
$$

Since by (3.4)

$$
\int_{0}^{t} \operatorname{Re}\langle\ddot{u}, \dot{u}\rangle \mathrm{d} s=\frac{1}{2} \int_{0}^{t}\left(\|\dot{u}\|_{H}^{2}\right)^{\cdot} \mathrm{d} s=\frac{1}{2}\|\dot{u}(t)\|_{H}^{2}-\frac{1}{2}\|\dot{u}(0)\|_{H}^{2},
$$

it follows by Young's inequality that

$$
\begin{aligned}
& \frac{1}{\alpha} \int_{0}^{t}\|f\|_{V^{\prime}}^{2} \mathrm{~d} s+\frac{\alpha}{4} \int_{0}^{t}\|\dot{u}\|_{V}^{2} \mathrm{~d} s \geq \int_{0}^{t}\|f\|_{V^{\prime}}\|\dot{u}\|_{V} \mathrm{~d} s \geq \int_{0}^{t} \operatorname{Re}\langle f, \dot{u}\rangle \mathrm{d} s \\
& \quad=\frac{1}{2}\|\dot{u}(t)\|_{H}^{2}-\frac{1}{2}\|\dot{u}(0)\|_{H}^{2}+\int_{0}^{t} \operatorname{Re} \mathfrak{b}(s, \dot{u}, \dot{u}) \mathrm{d} s+\int_{0}^{t} \operatorname{Re} \mathfrak{a}(s, u, \dot{u}) \mathrm{d} s \\
& \quad \geq-\frac{1}{2}\|\dot{u}(0)\|_{H}^{2}+\alpha \int_{0}^{t}\|\dot{u}\|_{V}^{2} \mathrm{~d} s-M \int_{0}^{t}\|u\|_{V}\|\dot{u}\|_{V} \mathrm{~d} s \\
& \quad \geq-\frac{1}{2}\|\dot{u}(0)\|_{H}^{2}+\frac{3 \alpha}{4} \int_{0}^{t}\|\dot{u}\|_{V}^{2} \mathrm{~d} s-\frac{M^{2}}{\alpha} \int_{0}^{t}\|u\|_{V}^{2} \mathrm{~d} s .
\end{aligned}
$$

Here we used coercivity of $\mathfrak{b}$ and $V$-boundedness of $\mathfrak{a}$. Therefore, by Lemma 3.2 , we have

$$
\begin{aligned}
\frac{1}{\alpha} \int_{0}^{t}\|f\|_{V^{\prime}}^{2} \mathrm{~d} s+\frac{1}{2}\|\dot{u}(0)\|_{H}^{2} \geq & \frac{\alpha}{2} \int_{0}^{t}\|\dot{u}\|_{V}^{2} \mathrm{~d} s-\frac{M^{2}}{\alpha} \int_{0}^{t}\|u\|_{V}^{2} \mathrm{~d} s \\
\geq & \left(\frac{\alpha}{2}-\frac{t^{2}\left(2 M^{2}+\alpha\right)}{\alpha}\right) \int_{0}^{t}\|\dot{u}\|_{V}^{2} \mathrm{~d} s \\
& -t\left(\frac{2 M^{2}+\alpha}{\alpha}\right)\|u(0)\|_{V}^{2}+\frac{1}{2} \int_{0}^{t}\|u\|_{V}^{2} \mathrm{~d} s .
\end{aligned}
$$

where we choose $t$ such that $\frac{\alpha}{2}>\frac{t^{2}\left(2 M^{2}+\alpha\right)}{\alpha}$. Finally, since

$$
\ddot{u}(s)=f(s)-\mathcal{A} \dot{u}(s)-\mathcal{B} u(s) \quad s \text {-a.e. }
$$


we obtain that

$$
\|\ddot{u}(s)\|_{V^{\prime}}^{2} \leq 3\|f(s)\|_{V^{\prime}}^{2}+3 M\|\dot{u}(s)\|_{V^{\prime}}^{2}+3 M\|u(s)\|_{V}^{2} \quad s \text {-a.e. }
$$

This together with (3.9) ends the proof of the proposition when $T$ is such that

$$
\frac{\alpha}{2}>\frac{T^{2}\left(2 M^{2}+\alpha\right)}{\alpha} .
$$

\section{Maximal Regularity for the Damped Wave Equation in $H$}

Let $V, H$ be separable Hilbert spaces such that $V \underset{d}{\hookrightarrow} H$ and let

$$
\mathfrak{a}, \mathfrak{b}:[0, T] \times V \times V \rightarrow \mathbb{K}
$$

be closed non-autonomous sesquilinear forms on which we impose the following conditions. Each can be written as the sum of two non-autonomous forms

$\mathfrak{a}(t, u, v)=\mathfrak{a}_{1}(t, u, v)+\mathfrak{a}_{2}(t, u, v), \quad \mathfrak{b}(t, u, v)=\mathfrak{b}_{1}(t, u, v)+\mathfrak{b}_{2}(t, u, v) \quad u, v \in V$

where

$$
\mathfrak{a}_{1}, \mathfrak{b}_{1}:[0, T] \times V \times V \rightarrow \mathbb{K}
$$

satisfy the following assumptions

a) $\left|\mathfrak{a}_{1}(t, u, v)\right| \leq M\|u\|_{V}\|v\|_{V}$ for all $u, v \in V, t \in[0, T]$;

b) $\mathfrak{a}_{1}(t, u, u) \geq \alpha\|u\|_{V}^{2}$ for all $u \in V, t \in[0, T]$ with $\alpha>0$;

c) $\mathfrak{a}_{1}(t, u, v)=\overline{\mathfrak{a}_{1}(t, v, u)}$ for all $u, v \in V, t \in[0, T]$;

d) $\mathfrak{a}_{1}$ is piecewise Lipschitz-continuous; i.e., there exist $0=\tau_{0}<\tau_{1}<\cdots<$ $\tau_{n}=T$ such that

$$
\left|\mathfrak{a}_{1}(t, u, v)-\mathfrak{a}_{1}(s, u, v)\right| \leq \dot{M}|t-s|\|u\|_{V}\|v\|_{V}
$$

for all $u, v \in V, s, t \in\left[\tau_{i-1}, \tau_{i}\right], i \in\{1, \ldots, n\}$,

and similarly for $\mathfrak{b}_{1}$. Of course we may choose the same constants $M, \dot{M}$ and $\alpha$ for both forms $\mathfrak{a}_{1}$ and $\mathfrak{b}_{1}$. We may also choose that same sub-intervals $0=\tau_{0}<$ $\tau_{1}<\cdots<\tau_{n}=T$ for both forms.

The non-autonomous forms

$$
\mathfrak{a}_{2}, \mathfrak{b}_{2}:[0, T] \times V \times V \rightarrow \mathbb{K}
$$

are measurable and satisfy

e) $\left|\mathfrak{a}_{2}(t, u, v)\right| \leq M\|u\|_{V}\|v\|_{H}$ for all $u, v \in V, t \in[0, T]$, 
and similarly for $\mathfrak{b}_{2}$.

Note that by Lemma 2.1 , if $\mathfrak{c}$ is a Lipschitz form on $[0, T]$, we may define its derivative $\dot{\mathfrak{c}}(t, .,$.$) and we have$

$$
|\dot{\mathfrak{c}}(t, u, v)| \leq \dot{M}\|u\|_{V}\|v\|_{V}, u, v \in V
$$

for some constant $\dot{M}$. We shall use this estimate for $\mathfrak{c}=\mathfrak{a}_{1}$ and for $\mathfrak{c}=\mathfrak{b}_{1}$ on sub-intervals of $[0, T]$ where these forms are supposed to be Lipschitz.

Let us denote by $\mathcal{A}(t)$ and $\mathcal{B}(t)$ the operators given by $\langle\mathcal{A}(t) u, v\rangle=\mathfrak{a}(t, u, v)$ and $\langle\mathcal{B}(t) u, v\rangle=\mathfrak{b}(t, u, v)$ for all $u, v \in V$.

As in the previous section we consider the damped wave equation. Here we study the maximal regularity property in $H$ rather than in $V^{\prime}$. We introduce the maximal regularity space

$$
M R(V, V, H):=H^{1}(0, T ; V) \cap H^{2}(0, T ; H) .
$$

We have

Theorem 4.1. Let $\mathfrak{a}=\mathfrak{a}_{1}+\mathfrak{a}_{2}$ and $\mathfrak{b}=\mathfrak{b}_{1}+\mathfrak{b}_{2}$ be non-autonomous $V$-bounded and quasi-coercive forms satisfying the above properties a) $-e$ ). Then for every $u_{0}, u_{1} \in V$ and $f \in L^{2}(0, T ; H)$, there exists a unique solution $u \in M R(V, V, H)$ of the non-autonomous second order Cauchy problem

$$
\left\{\begin{array}{l}
\ddot{u}(t)+\mathcal{B}(t) \dot{u}(t)+\mathcal{A}(t) u(t)=f(t) \quad \text { t-a.e. } \\
u(0)=u_{0}, \dot{u}(0)=u_{1}
\end{array}\right.
$$

Moreover $\dot{u}(t) \in V$ for all $t \in[0, T]$.

For a related result see Lions [Lio61, p. 155]. However the result proved there is restricted to $u_{1}=0$ and assumes $f, f^{\prime} \in L^{2}(0, T, H)$. Our proof resembles that of Theorem 3.1 and uses similar ideas as in Lions [Lio61].

We use the following lemma for the proof of Theorem 4.1.

Lemma 4.2. Suppose that the forms $\mathfrak{a}_{1}$ and $\mathfrak{b}_{1}$ are Lipschitz continuous on $[0, T]$. Let $v \in H^{2}(0, T ; V)$ and $\epsilon>0$. Then

(i) $\int_{0}^{T} e^{-\lambda t} \operatorname{Re} \mathfrak{b}_{1}(t, \dot{v}, \ddot{v}) \mathrm{d} t=\frac{\lambda}{2} \int_{0}^{T} e^{-\lambda t} \mathfrak{b}_{1}(t, \dot{v}, \dot{v}) \mathrm{d} t-\frac{1}{2} \int_{0}^{T} e^{-\lambda t} \dot{\mathfrak{b}}_{1}(t, \dot{v}, \dot{v}) \mathrm{d} t$ $+\frac{1}{2} e^{-\lambda T} \mathfrak{b}_{1}(T, \dot{v}(T), \dot{v}(T))-\frac{1}{2} \mathfrak{b}_{1}(0, \dot{v}(0), \dot{v}(0))$.

(ii) $\int_{0}^{T} e^{-\lambda t} \operatorname{Re} \mathfrak{a}_{1}(t, v, \ddot{v}) \mathrm{d} t=\frac{\lambda}{2} e^{-\lambda T} \mathfrak{a}_{1}(T, v(T), v(T))-\frac{\lambda}{2} \mathfrak{a}_{1}(0, v(0), v(0))$

$$
\begin{aligned}
& +e^{-\lambda T} \operatorname{Re} \mathfrak{a}_{1}(T, v(T), \dot{v}(T))-\operatorname{Re} \mathfrak{a}_{1}(0, v(0), \dot{v}(0)) \\
& +\frac{\lambda^{2}}{2} \int_{0}^{T} e^{-\lambda t} \mathfrak{a}_{1}(t, v, v) \mathrm{d} t-\frac{\lambda}{2} \int_{0}^{T} e^{-\lambda t} \dot{\mathfrak{a}}_{1}(t, v, v) \mathrm{d} t \\
& -\int_{0}^{T} e^{-\lambda t} \operatorname{Re} \dot{\mathfrak{a}}_{1}(t, v, \dot{v}) \mathrm{d} t-\int_{0}^{T} e^{-\lambda t} \mathfrak{a}_{1}(t, \dot{v}, \dot{v}) \mathrm{d} t .
\end{aligned}
$$

(iii) $\int_{0}^{T} e^{-\lambda t} \operatorname{Re} \mathfrak{b}_{1}(t, \dot{v}, \ddot{v}) \mathrm{d} t+\int_{0}^{T} e^{-\lambda t} \operatorname{Re} \mathfrak{a}_{1}(t, v, \ddot{v}) \mathrm{d} t$

$$
\geq \frac{1}{2}(\alpha \lambda-2 \dot{M}-2 M) \int_{0}^{T} e^{-\lambda t}\|\dot{v}\|_{V}^{2} \mathrm{~d} t
$$




$$
\begin{aligned}
& +\left(\frac{\lambda}{2}(\alpha \lambda-\dot{M})-\frac{\dot{M}^{2}}{2}\right) \int_{0}^{T} e^{-\lambda t}\|v\|_{V}^{2} \mathrm{~d} t \\
& +\frac{1}{2} e^{-\lambda T}\left[(\alpha-\epsilon)\|\dot{v}(T)\|_{V}^{2}+\left(\lambda \alpha-\frac{\dot{M}^{2}}{\epsilon}\right)\|v(T)\|_{V}^{2}\right] \\
& -\frac{1}{2} \mathfrak{b}_{1}(0, \dot{v}(0), \dot{v}(0))-\frac{\lambda}{2} \mathfrak{a}_{1}(0, v(0), v(0))-\operatorname{Re} \mathfrak{a}_{1}(0, v(0), \dot{v}(0)) .
\end{aligned}
$$

Proof. The proof of (i) and (ii) is based on Lemma 2.1 and the product rule.

Part (i) is a direct consequence of the formulae

$\left(e^{-\lambda t} \mathfrak{b}_{1}(t, \dot{v}, \dot{v})\right)^{\cdot}=-\lambda e^{-\lambda t} \mathfrak{b}_{1}(t, \dot{v}, \dot{v})+e^{-\lambda t} \dot{\mathfrak{b}}_{1}(t, \dot{v}, \dot{v})+2 e^{-\lambda t} \operatorname{Re} \mathfrak{b}_{1}(t, \dot{v}, \ddot{v})$.

For (ii) we first calculate the following derivatives

$$
\begin{aligned}
\operatorname{Re}\left(e^{-\lambda t} \mathfrak{a}_{1}(t, v, v)\right)^{\cdot}= & -\lambda e^{-\lambda t} \mathfrak{a}_{1}(t, v, v)+e^{-\lambda t} \operatorname{Re} \dot{\mathfrak{a}}_{1}(t, v, v) \\
& +2 e^{-\lambda t} \operatorname{Re} \mathfrak{a}_{1}(t, v, \dot{v}) \\
\operatorname{Re}\left(e^{-\lambda t} \mathfrak{a}_{1}(t, v, \dot{v})\right)^{\cdot}= & -\lambda e^{-\lambda t} \operatorname{Re} \mathfrak{a}_{1}(t, v, \dot{v})+e^{-\lambda t} \operatorname{Re} \dot{\mathfrak{a}}_{1}(t, v, \dot{v}) \\
& +e^{-\lambda t} \mathfrak{a}_{1}(t, \dot{v}, \dot{v})+e^{-\lambda t} \operatorname{Re} \mathfrak{a}_{1}(t, v, \ddot{v})
\end{aligned}
$$

then we multiply the first equation by $\frac{\lambda}{2}$ and add the second equation. Now (ii) follows by integration over $t$ from 0 to $T$.

For (iii) we add (i) and (ii) and use coercivity of $\mathfrak{a}_{1}, \mathfrak{b}_{1}$ and $V$-boundedness of $\mathfrak{a}_{1}, \dot{\mathfrak{a}}_{1}, \mathfrak{b}_{1}, \dot{\mathfrak{b}}_{1}$. Thus

$$
\begin{aligned}
\int_{0}^{T} e^{-\lambda t} & \operatorname{Re} \mathfrak{b}_{1}(t, \dot{v}, \ddot{v}) \mathrm{d} t+\int_{0}^{T} e^{-\lambda t} \operatorname{Re} \mathfrak{a}_{1}(t, v, \ddot{v}) \mathrm{d} t \\
\geq & \frac{1}{2}(\alpha \lambda-\dot{M}-2 M) \int_{0}^{T} e^{-\lambda t}\|\dot{v}\|_{V}^{2} \mathrm{~d} t \\
& +\frac{\lambda}{2}(\alpha \lambda-\dot{M}) \int_{0}^{T} e^{-\lambda t}\|v\|_{V}^{2} \mathrm{~d} t-\dot{M} \int_{0}^{T} e^{-\lambda t}\|v\|_{V}\|\dot{v}\|_{V} \mathrm{~d} t \\
& +\frac{1}{2} e^{-\lambda T}\left[\alpha\|\dot{v}(T)\|_{V}^{2}+\lambda \alpha\|v(T)\|_{V}^{2}-2 M\|v(T)\|_{V}\|\dot{v}(T)\|_{V}\right] \\
& -\frac{1}{2} \mathfrak{b}_{1}(0, \dot{v}(0), \dot{v}(0))-\frac{\lambda}{2} \mathfrak{a}_{1}(0, v(0), v(0))-\operatorname{Re} \mathfrak{a}_{1}(0, v(0), \dot{v}(0)) .
\end{aligned}
$$

We apply Young's inequality and see that the last term is bounded from below by

$$
\begin{aligned}
& \frac{1}{2}(\alpha \lambda-2 \dot{M}-2 M) \int_{0}^{T} e^{-\lambda t}\|\dot{v}\|_{V}^{2} \mathrm{~d} t+\left(\frac{\lambda}{2}(\alpha \lambda-\dot{M})-\frac{\dot{M}^{2}}{2}\right) \int_{0}^{T} e^{-\lambda t}\|v\|_{V}^{2} \mathrm{~d} t \\
& \quad+\frac{1}{2} e^{-\lambda T}\left[(\alpha-\epsilon)\|\dot{v}(T)\|_{V}^{2}+\left(\lambda \alpha-\frac{\dot{M}^{2}}{\epsilon}\right)\|v(T)\|_{V}^{2}\right] \\
& \quad-\frac{1}{2} \mathfrak{b}_{1}(0, \dot{v}(0), \dot{v}(0))-\frac{\lambda}{2} \mathfrak{a}_{1}(0, v(0), v(0))-\operatorname{Re} \mathfrak{a}_{1}(0, v(0), \dot{v}(0))
\end{aligned}
$$

for $\epsilon>0$.

Proof of Theorem 4.1. Uniqueness follows from Theorem 3.1 and we only need to prove existence of a solution. As in the proof of Theorem 3.1 we may assume that the forms $\mathfrak{a}$ and $\mathfrak{b}$ are both coercive (see (3.6)).

1- Lipschitz-continuous forms. Suppose first that the forms $\mathfrak{a}_{1}$ and $\mathfrak{b}_{1}$ are Lipschitz-continuous on $[0, T]$. 
We define the Hilbert space

$$
\mathcal{H}:=\left\{u \in H^{2}(0, T ; H) \cap H^{1}(0, T ; V): u(0), \dot{u}(0), \dot{u}(T) \in V\right\}
$$

with norm $\|u\|_{\mathcal{H}}$ given by

$$
\|u\|_{\mathcal{H}}^{2}:=\|\ddot{u}\|_{L^{2}(0, T ; H)}^{2}+\|u\|_{H^{1}(0, T ; V)}^{2}+\|u(0)\|_{V}^{2}+\|\dot{u}(0)\|_{V}^{2}+\|\dot{u}(T)\|_{V}^{2}
$$

and the pre-Hilbert space $\mathcal{V}:=H^{2}(0, T ; V)$ with norm $\|\cdot\|_{\mathcal{V}}:=\|\cdot\|_{\mathcal{H}}$. Next we define the sesquilinear form $E: \mathcal{H} \times \mathcal{V} \rightarrow \mathbb{C}$ by

$$
\begin{aligned}
E(u, v):= & \int_{0}^{T} e^{-\lambda t}(\ddot{u} \mid \ddot{v})_{H} \mathrm{~d} t+\int_{0}^{T} e^{-\lambda t} \mathfrak{b}(t, \dot{u}, \ddot{v}) \mathrm{d} t+\int_{0}^{T} e^{-\lambda t} \mathfrak{a}(t, u, \ddot{v}) \mathrm{d} t \\
& +\eta(\dot{u}(0) \mid \dot{v}(0))_{V}+\eta(u(0) \mid v(0))_{V},
\end{aligned}
$$

where $\lambda$ and $\eta$ are positive parameters. Later on, we will choose them to be large enough. For $u_{0}, u_{1} \in V$ and $f \in L^{2}(0, T ; H)$, we define $F: \mathcal{V} \rightarrow \mathbb{C}$ by

$$
F(v):=\int_{0}^{T} e^{-\lambda t}(f \mid \ddot{v})_{H} \mathrm{~d} t+\eta\left(u_{1} \mid \dot{v}(0)\right)_{V}+\eta\left(u_{0} \mid v(0)\right)_{V}
$$

We proceed as in the proof of Theorem 3.1. Suppose for a moment that

1) $E(., v) \in \mathcal{H}^{\prime}$ and $F \in \mathcal{V}^{\prime}$;

2) $E$ is coercive; i.e., there exists a $C>0$ such that $|E(v, v)| \geq C\|v\|_{\mathcal{H}}^{2}$ for all $v \in \mathcal{V}$.

Then by Lions's representation theorem there exists $u \in \mathcal{H}$ such that

$$
E(u, v)=F(v)
$$

for all $v \in \mathcal{V}$. For arbitrary $w \in V$ and $\psi \in \mathcal{D}(0, T)$ we take $v(t)=\int_{0}^{t} \int_{0}^{s} \psi(r) \mathrm{d} r \mathrm{~d} s w$. It follows from (4.3) that

$$
\ddot{u}(t)+\mathcal{B}(t) \dot{u}(t)+\mathcal{A}(t) u(t)=f(t)
$$

in $L^{2}\left(0, T ; V^{\prime}\right)$. This identity applied to (4.3) implies that

$$
\eta(\dot{u}(0) \mid \dot{v}(0))_{V}+\eta(u(0) \mid v(0))_{V}=\eta\left(u_{1} \mid \dot{v}(0)\right)_{V}+\eta\left(u_{0} \mid v(0)\right)_{V}
$$

for all $v \in \mathcal{V}$. Hence $u(0)=u_{0}$ and $\dot{u}(0)=u_{1}$. This means that $u \in M R(V, V, H)$ is a solution of (4.2).

It remain to prove properties 1) and 2). Again, 1) is obvious and we focus on 2). Let $v \in \mathcal{V}$. For $\epsilon \in(0, \alpha)$ set

$$
\begin{aligned}
R:= & \eta\|\dot{v}(0)\|_{V}^{2}+\eta\|v(0)\|_{V}^{2}+\frac{1}{2} e^{-\lambda T}\left[(\alpha-\epsilon)\|\dot{v}(T)\|_{V}^{2}+\left(\lambda \alpha-\frac{\dot{M}^{2}}{\epsilon}\right)\|v(T)\|_{V}^{2}\right] \\
& -\frac{1}{2} \mathfrak{b}_{1}(0, \dot{v}(0), \dot{v}(0))-\frac{\lambda}{2} \mathfrak{a}_{1}(0, v(0), v(0))-\operatorname{Re} \mathfrak{a}_{1}(0, v(0), \dot{v}(0)) .
\end{aligned}
$$

By the $V$-boundedness of $\mathfrak{a}_{1}$ and $\mathfrak{b}_{1}$ we have

$$
R \geq \frac{1}{2} e^{-\lambda T}\left[(\alpha-\epsilon)\|\dot{v}(T)\|_{V}^{2}+\left(\lambda \alpha-\frac{\dot{M}^{2}}{\epsilon}\right)\|v(T)\|_{V}^{2}\right]+\left(\eta-\frac{M}{2}\right)\|\dot{v}(0)\|_{V}^{2}
$$




$$
+\left(\eta-\frac{\lambda M}{2}\right)\|v(0)\|_{V}^{2}-M\|\dot{v}(0)\|_{V}\|v(0)\|_{V} .
$$

Young's inequality yields

$$
R \geq C_{1}\left[\|\dot{v}(T)\|_{V}^{2}+\|v(T)\|_{V}^{2}+\|\dot{v}(0)\|_{V}^{2}+\|v(0)\|_{V}^{2}\right]
$$

for some $C_{1}>0$ provided $\lambda$ and $\eta$ are sufficiently large. Now

$$
\begin{aligned}
\operatorname{Re} E(v, v)= & \int_{0}^{T} e^{-\lambda t}\|\ddot{v}\|_{H}^{2} \mathrm{~d} t+\int_{0}^{T} e^{-\lambda t} \operatorname{Re} \mathfrak{b}_{1}(t, \dot{v}, \ddot{v}) \mathrm{d} t \\
& +\int_{0}^{T} e^{-\lambda t} \operatorname{Re} \mathfrak{b}_{2}(t, \dot{v}, \ddot{v}) \mathrm{d} t+\int_{0}^{T} e^{-\lambda t} \operatorname{Re} \mathfrak{a}_{1}(t, v, \ddot{v}) \mathrm{d} t \\
& +\int_{0}^{T} e^{-\lambda t} \operatorname{Re} \mathfrak{a}_{2}(t, v, \ddot{v}) \mathrm{d} t+\eta(\dot{v}(0) \mid \dot{v}(0))_{V}+\eta(v(0) \mid v(0))_{V}
\end{aligned}
$$

We apply assertion (iii) of Lemma 4.2, it follows that

$$
\begin{aligned}
\operatorname{Re} E(v, v) \geq & \int_{0}^{T} e^{-\lambda t}\|\ddot{v}\|_{H}^{2} \mathrm{~d} t+\int_{0}^{T} e^{-\lambda t} \operatorname{Re} \mathfrak{b}_{2}(t, \dot{v}, \ddot{v}) \mathrm{d} t \\
& +\int_{0}^{T} e^{-\lambda t} \operatorname{Re} \mathfrak{a}_{2}(t, v, \ddot{v}) \mathrm{d} t \\
& +\frac{1}{2}(\alpha \lambda-2 \dot{M}-2 M) \int_{0}^{T} e^{-\lambda t}\|\dot{v}\|_{V}^{2} \mathrm{~d} t \\
& +\frac{1}{2}(\lambda(\alpha \lambda-\dot{M})-\dot{M}) \int_{0}^{T} e^{-\lambda t}\|v\|_{V}^{2} \mathrm{~d} t+R
\end{aligned}
$$

Thus $V$-boundedness of $\mathfrak{a}_{2}$ and $\mathfrak{b}_{2}$ and Young's inequality yield

$$
\operatorname{Re} E(v, v) \geq C\|v\|_{\mathcal{H}}^{2},
$$

for some $C>0$ provided that $\lambda$ and $\eta$ are sufficiently large. This proves 2).

Finally, we have seen that the unique solution $u$ satisfies $\dot{u}(T) \in V$ but we may replace in the previous arguments $[0, T]$ by $[0, t]$ for any fixed $t \in(0, T)$ and obtain $\dot{u}(t) \in V$.

2- Piecewise Lipschitz-continuous forms. Suppose now that the forms $\mathfrak{a}_{1}$ and $\mathfrak{b}_{1}$ satisfy assumption d). We may replace in the first step the interval $[0, T]$ by $\left[\tau_{i-1}, \tau_{i}\right]$. There exists a solution $u^{i} \in H^{1}\left(\tau_{i-1}, \tau_{i} ; V\right) \cap H^{2}\left(\tau_{i-1}, \tau_{i} ; H\right)$ of the equation

$$
\ddot{v}(t)+\mathcal{B}(t) \dot{u}(t)+\mathcal{A}(t) u(t)=f(t) \text { a.e. } t \in\left[\tau_{i-1}, \tau_{i}\right],
$$

with prescribed $u^{i}\left(\tau_{i-1}\right), \dot{u}^{i}\left(\tau_{i-1}\right)$ in $V$. We also know from the previous step that $u^{i}\left(\tau_{i}\right), \dot{u}^{i}\left(\tau_{i}\right) \in V$. Now we can solve the previous equation on $\left[\tau_{i}, \tau_{i+1}\right]$ and obtain a solution $u^{i+1}$ such that $u^{i+1}\left(\tau_{i}\right)=u^{i}\left(\tau_{i}\right)$ and $\dot{u}^{i+1}\left(\tau_{i}\right)=\dot{u}^{i}\left(\tau_{i}\right)$. We define $u$ on $[0, T]$ by $u=u^{i}$ on $\left[\tau_{i-1}, \tau_{i}\right]$. It is easy to check that $u \in M R(V, V, H)$ and $u$ is a solution to (4.2). This finishes the proof of the theorem. 


\section{The Wave Equation}

Let $H, V$ be Hilbert spaces such that $V \stackrel{d}{\hookrightarrow} H$. Suppose $\mathfrak{a}:[0, T] \times V \times V \rightarrow$ $\mathbb{C}$ is a Lipschitz-continuous, symmetric, V-bounded and quasi-coercive nonautonomous form. We denote again by $\mathcal{A}(t)$ the operator associated with $\mathfrak{a}(t)$ on $V^{\prime}$ and by $A(t)$ the part of $\mathcal{A}(t)$ in $H$.

We introduce the maximal regularity space

$$
M R\left(V, H, V^{\prime}\right):=L^{2}(0, T ; V) \cap H^{1}(0, T ; H) \cap H^{2}\left(0, T ; V^{\prime}\right)
$$

for the second order Cauchy problem. We have the following result.

Theorem 5.1. There exists a unique solution $u \in M R\left(V, H, V^{\prime}\right)$ of the nonautonomous second order Cauchy problem

$$
\left\{\begin{array}{l}
\ddot{u}(t)+\mathcal{A}(t) u(t)=f(t) \quad t \text {-a.e. } \\
u(0)=u_{0}, \dot{u}(0)=u_{1}
\end{array}\right.
$$

for every $u_{0} \in V, u_{1} \in H$ and $f \in L^{2}(0, T ; H)$. Moreover $u(t) \in V$ for all $t \in[0, T]$.

Note that by [DL88, p. 579], for every $u \in M R\left(V, H, V^{\prime}\right), \dot{u}$ can be viewed as a continuous function from $[0, T]$ into the interpolation space $\left(H, V^{\prime}\right)_{\frac{1}{2}}$. In particular, $\dot{u}(0)$ is well defined and $\dot{u}(0) \in V^{\prime}$.

We start with the following lemma. Here $\dot{\mathfrak{a}}(t, .,$.$) denotes the derivative of$ $t \mapsto \mathfrak{a}(t, .,)$.

Lemma 5.2. Let $v \in H^{2}(0, T ; V)$ with $\dot{v}(T)=0$. Then

$$
\begin{aligned}
\text { (i) } \int_{0}^{T} e^{-\lambda t} \operatorname{Re}(\ddot{v} \mid \dot{v})_{H} \mathrm{~d} t= & \frac{\lambda}{2} \int_{0}^{T} e^{-\lambda t}\|\dot{v}\|_{H}^{2} \mathrm{~d} t-\frac{1}{2}\|\dot{v}(0)\|_{H}^{2} \\
\text { (ii) } \int_{0}^{T} e^{-\lambda t} \operatorname{Re} \mathfrak{a}(t, v, \dot{v}) \mathrm{d} t= & \frac{\lambda}{2} \int_{0}^{T} e^{-\lambda t} \mathfrak{a}(t, v, v) \mathrm{d} t-\frac{1}{2} \int_{0}^{T} e^{-\lambda t} \dot{\mathfrak{a}}(t, v, v) \mathrm{d} t \\
& +\frac{1}{2} \mathfrak{a}(T, v(T), v(T))-\frac{1}{2} \mathfrak{a}(0, v(0), v(0))
\end{aligned}
$$

Proof. For the first part we calculate the formula

$$
\left(e^{-\lambda t}\|\dot{v}\|_{H}^{2}\right)^{\cdot}=-\lambda e^{-\lambda t}\|\dot{v}\|_{H}^{2}+2 e^{-\lambda t} \operatorname{Re}(\ddot{v}, \dot{v})_{H} .
$$

For (ii) we use Lemma 2.1 and the product rule to obtain

$$
\left(e^{-\lambda t} \mathfrak{a}(t, v, v)\right)^{\cdot}=-\lambda e^{-\lambda t} \mathfrak{a}(t, v, v)+2 e^{-\lambda t} \operatorname{Re} \mathfrak{a}(t, v, \dot{v})+e^{-\lambda t} \dot{\mathfrak{a}}(t, v, v) .
$$

Now the Lemma follows by integrating over $t$.

Proof of Theorem 5.1. First we prove existence of a solution. We define the Hilbert space $\mathcal{H}:=\left\{u \in L^{2}(0, T ; V) \cap H^{1}(0, T ; H): u(0), u(T) \in V\right\}$ with norm $\|u\|_{\mathcal{H}}$ such that $\|u\|_{\mathcal{H}}^{2}:=\|u\|_{L^{2}(0, T ; V)}^{2}+\|\dot{u}\|_{L^{2}(0, T ; H)}^{2}+\|u(0)\|_{V}^{2}+\|u(T)\|_{V}^{2}$ and the pre-Hilbert space $\mathcal{V}:=\left\{v \in H^{2}(0, T ; V): \dot{v}(T)=0\right\}$ with norm $\|.\|_{\mathcal{V}}:=\|\cdot\|_{\mathcal{H}}$. Further we define $E: \mathcal{H} \times \mathcal{V} \rightarrow \mathbb{C}$ by

$$
E(u, v):=-\int_{0}^{T}\left(\dot{u} \mid\left(e^{-\lambda t} \dot{v}\right)^{\cdot}\right)_{H} \mathrm{~d} t
$$




$$
+\int_{0}^{T} e^{-\lambda t} \mathfrak{a}(t, u, \dot{v}) \mathrm{d} t+\mathfrak{a}(0, u(0), v(0))
$$

and for $u_{0} \in V, u_{1} \in H$ and $f \in L^{2}\left(0, T ; V^{\prime}\right)$ we define $F: \mathcal{V} \rightarrow \mathbb{C}$ by

$$
F(v):=\int_{0}^{T} e^{-\lambda t}\langle f, \dot{v}\rangle \mathrm{d} t+\mathfrak{a}\left(0, u_{0}, v(0)\right)+\left(u_{1} \mid \dot{v}(0)\right)_{H} .
$$

As in the previous sections, we use Lions's representation Theorem. Suppose that the assumptions of Lions's Theorem are satisfied. Then there exists a $u \in \mathcal{H}$ such that

$$
E(u, v)=F(v)
$$

for all $v \in \mathcal{V}$. For the particular choice of $v(t):=\psi(t) w$ where $\psi \in \mathcal{D}(0, T)$ and $w \in V$ we obtain from (5.2) that

$$
\int_{0}^{T}\langle\dot{u}, w\rangle\left(e^{-\lambda t} \dot{\psi}(t)\right)^{\cdot} \mathrm{d} t=\int_{0}^{T}\langle f-\mathcal{A} u, w\rangle e^{-\lambda t} \dot{\psi}(t) \mathrm{d} t .
$$

This implies that $\dot{u} \in H^{1}\left(0, T ; V^{\prime}\right)$, hence $u \in M R\left(V, H, V^{\prime}\right)$ and that

$$
\ddot{u}(t)+\mathcal{A}(t) u(t)=f(t) \quad t \text {-a.e. }
$$

Following the proof of Lemma 1 and Theorem 2 in [DL88, p. 571 and 575] we can integrate by parts in the first term of $E(u, v)$ to obtain

$$
\begin{aligned}
E(u, v)= & \langle\dot{u}(0), \dot{v}(0)\rangle+\int_{0}^{T} e^{-\lambda t}\langle\ddot{u}, \dot{v}\rangle \mathrm{d} t \\
& +\int_{0}^{T} e^{-\lambda t} \mathfrak{a}(t, u, \dot{v}) \mathrm{d} t+\mathfrak{a}(0, u(0), v(0)) \\
= & \int_{0}^{T} e^{-\lambda t}\langle f, \dot{v}\rangle \mathrm{d} t+\mathfrak{a}\left(0, u_{0}, v(0)\right)+\left(u_{1} \mid \dot{v}(0)\right)_{H}
\end{aligned}
$$

where we used the identity (5.2). This together with (5.3) implies that

$$
\langle\dot{u}(0), \dot{v}(0)\rangle+\mathfrak{a}(0, u(0), v(0))=\mathfrak{a}\left(0, u_{0}, v(0)\right)+\left(u_{1} \mid \dot{v}(0)\right)_{H} .
$$

Since $v \in \mathcal{V}$ was arbitrary this shows that $u(0)=u_{0}$ and $\dot{u}(0)=u_{1}$.

Next we check the assumptions of Theorem 2.4. Assumption 1) is again easy to verify. Let $v \in \mathcal{V}$, then integration by parts yields to

$$
\begin{aligned}
|E(v, v)| \geq \operatorname{Re} E(v, v)= & \|\dot{v}(0)\|_{H}^{2}+\int_{0}^{T} e^{-\lambda t} \operatorname{Re}(\ddot{v} \mid \dot{v})_{H} \mathrm{~d} t \\
& +\int_{0}^{T} e^{-\lambda t} \operatorname{Re} \mathfrak{a}(t, v, \dot{v}) \mathrm{d} t+\mathfrak{a}(0, v(0), v(0)) .
\end{aligned}
$$

Thus Lemma 5.2 applied to the first and second integral and Young's inequality shows that

$$
\operatorname{Re} E(v, v) \geq \frac{1}{2}\|\dot{v}(0)\|_{H}^{2}+\frac{\lambda}{2} \int_{0}^{T} e^{-\lambda t}\|\dot{v}\|_{H}^{2} \mathrm{~d} t+\frac{\lambda}{2} \int_{0}^{T} e^{-\lambda t} \mathfrak{a}(t, v, v) \mathrm{d} t
$$




$$
\begin{aligned}
& -\frac{1}{2} \int_{0}^{T} e^{-\lambda t} \dot{\mathfrak{a}}(t, v, v) \mathrm{d} t+\frac{1}{2} \mathfrak{a}(T, v(T), v(T))+\frac{1}{2} \mathfrak{a}(0, v(0), v(0)) \\
\geq & C\|v\|_{\mathcal{H}}^{2}
\end{aligned}
$$

for some $C>0$ if $\lambda$ is large enough. Note that we can choose $C$ depending only on the coercivity, $V$-boundedness, Lipschitz constant of the form and on $T$.

Uniqueness: Let $u \in M R\left(V, H, V^{\prime}\right)$ be a solution of (5.1) where $f=0$ and $u_{0}=u_{1}=0$. We have to show that $u=0$. Fix $r \in[0, T]$ and define $v_{r}(t):=$ $\int_{t}^{T} \mathbb{1}_{[0, r]} u(s) \mathrm{d} s$. Then $v_{r} \in H^{1}(0, T ; V)$ with $v_{r}(r)=0$ and $\dot{v}_{r}=-\mathbb{1}_{[0, r]} u$. We obtain

$$
\begin{aligned}
0 & =2 \int_{0}^{T} \operatorname{Re}\left\langle\ddot{u}, v_{r}\right\rangle \mathrm{d} t+2 \int_{0}^{T} \operatorname{Re} \mathfrak{a}\left(t, u, v_{r}\right) \mathrm{d} t \\
& =2 \int_{0}^{r} \int_{t}^{r} \operatorname{Re}\langle\ddot{u}(t), u(s)\rangle \mathrm{d} s \mathrm{~d} t-2 \int_{0}^{r} \operatorname{Re} \mathfrak{a}\left(t, \dot{v}_{r}, v_{r}\right) \mathrm{d} t \\
& =2 \int_{0}^{r} \int_{0}^{s} \operatorname{Re}\langle\ddot{u}(t), u(s)\rangle \mathrm{d} t \mathrm{~d} s-2 \int_{0}^{r} \operatorname{Re} \mathfrak{a}\left(t, \dot{v}_{r}, v_{r}\right) \mathrm{d} t \\
& =2 \int_{0}^{r} \operatorname{Re}\left\langle\int_{0}^{s} \ddot{u}(t) \mathrm{d} t, u(s)\right\rangle \mathrm{d} s-\int_{0}^{r}\left(\mathfrak{a}\left(t, v_{r}, v_{r}\right)\right) \cdot \dot{\mathfrak{a}}\left(t, v_{r}, v_{r}\right) \mathrm{d} t \\
& =2 \int_{0}^{r} \operatorname{Re}\langle\dot{u}, u\rangle \mathrm{d} s+\mathfrak{a}\left(0, v_{r}(0), v_{r}(0)\right)-\int_{0}^{r} \dot{\mathfrak{a}}\left(t, v_{r}, v_{r}\right) \mathrm{d} t \\
& \geq\|u(r)\|_{H}^{2}+\alpha\left\|v_{r}(0)\right\|_{V}^{2}-\dot{M} \int_{0}^{r}\left\|v_{r}\right\|_{V}^{2} \mathrm{~d} t .
\end{aligned}
$$

We set $w(r):=v_{r}(0)=\int_{0}^{r} u(s) \mathrm{d} s \in L^{2}(0, T ; V)$. Then $w(r)-w(t)=v_{r}(t)$ and

$$
\alpha\|w(r)\|_{V}^{2} \leq \dot{M} \int_{0}^{r}\|w(r)-w(t)\|_{V}^{2} \mathrm{~d} t \leq 2 r \dot{M}\|w(r)\|_{V}^{2}+2 \dot{M} \int_{0}^{r}\|w(t)\|_{V}^{2} \mathrm{~d} t .
$$

Let $0<r_{0}<\frac{\alpha}{2 \dot{M}}$ and set $C_{r_{0}}:=\alpha-2 r_{0} \dot{M}>0$, then for every $r \in\left[0, r_{0}\right]$ we have

$$
\|w(r)\|_{V}^{2} \leq 2 \dot{M} C_{r_{0}}^{-1} \int_{0}^{r}\|w(t)\|_{V}^{2} \mathrm{~d} t
$$

We conclude by Gronwall's lemma that $w(r)=0$ for all $r \in\left[0, r_{0}\right]$, hence $u=0$ on $\left[0, r_{0}\right]$. Now we may proceed inductively to obtain $u=0$ on $[0, T]$.

Remark 5.3. If we add a $V \times H$-bounded perturbation to $\mathfrak{a}$ as in Section 4, we can still prove existence in Theorem 5.1. But for the uniqueness we have to assume additionally that this perturbation is also $H \times V$-bounded.

Remark 5.4. Let $B(t)$ be bounded operators on $H$ with $\|B(t)\|_{\mathcal{L}(H)} \leq M_{B}$ for a.e. $t \in[0, T]$. We consider the wave equation

$$
\left\{\begin{array}{l}
\ddot{u}(t)+B(t) \dot{u}(t)+\mathcal{A}(t) u(t)=f(t) \quad t \text {-a.e. } \\
u(0)=u_{0}, \dot{u}(0)=u_{1}
\end{array}\right.
$$

Then for $u_{0} \in V, u_{1} \in H$ and $f \in L^{2}\left(0, T, V^{\prime}\right)$ there exists a solution $u \in$ $M R\left(V, H, V^{\prime}\right)$ to (5.4). The proof is the same as above, one has only to change $E(u, v)$ into

$$
E(u, v):=-\int_{0}^{T}\left(\dot{u} \mid\left(e^{-\lambda t} \dot{v}\right)^{\cdot}\right)_{H} \mathrm{~d} t
$$




$$
\begin{aligned}
& +\int_{0}^{T} e^{-\lambda t}(B(t) \dot{u} \mid \dot{v})_{H} \mathrm{~d} t \\
& +\int_{0}^{T} e^{-\lambda t} \mathfrak{a}(t, u, \dot{v}) \mathrm{d} t+\mathfrak{a}(0, u(0), v(0)) .
\end{aligned}
$$

The uniqueness of $u$ is however not clear except if the map $t \mapsto B(t)$ is Lipschitz. If this later condition is satisfied one can use similar ideas as in [DL88, p. 686] to prove uniqueness. The proof for uniqueness in Theorem 5.1 is similar to that of [DL88, p. 673].

\section{Applications}

In this section we give applications of our results. We consider two problems, one is linear and the second one is quasi-linear.

I) Laplacian with time dependent Robin boundary conditions.

Let $\Omega$ be a bounded domain of $\mathbb{R}^{d}$ with Lipschitz boundary $\Gamma$. Denote by $\sigma$ be the $(d-1)$-dimensional Hausdorff measure on $\Gamma$. Let

$$
\beta_{1}, \beta_{2}:[0, T] \times \Gamma \rightarrow \mathbb{R}
$$

be bounded measurable functions which are Lipschitz continuous w.r.t. the first variable, i.e.,

$$
\left|\beta_{i}(t, x)-\beta_{i}(s, x)\right| \leq M|t-s| \quad(i=1,2)
$$

for some constant $M$ and all $t, s \in[0, T], x \in \Gamma$. We consider the symmetric forms

$$
\mathfrak{a}, \mathfrak{b}:[0, T] \times H^{1}(\Omega) \times H^{1}(\Omega) \rightarrow \mathbb{R}
$$

defined by

$$
\mathfrak{a}(t, u, v)=\int_{\Omega} \nabla u \nabla v \mathrm{~d} x+\int_{\Gamma} \beta_{1}(t, .) u v \mathrm{~d} \sigma .
$$

and

$$
\mathfrak{b}(t, u, v)=\int_{\Omega} \nabla u \nabla v \mathrm{~d} x+\int_{\Gamma} \beta_{2}(t, .) u v \mathrm{~d} \sigma .
$$

respectively. The forms $\mathfrak{a}, \mathfrak{b}$ are $H^{1}(\Omega)$-bounded and quasi-coercive. The first statement follows readily from the continuity of the trace operator and the boundedness of $\beta$. The second one is a consequence of the inequality

$$
\int_{\Gamma}|u|^{2} \mathrm{~d} \sigma \leq \epsilon\|u\|_{H^{1}(\Omega)}^{2}+c_{\epsilon}\|u\|_{L^{2}(\Omega)}^{2},
$$

which is valid for all $\epsilon>0$ ( $c_{\epsilon}$ is a constant depending on $\epsilon$ ). Note that (6.4) is a consequence of compactness of the trace as an operator from $H^{1}(\Omega)$ into $L^{2}(\Gamma, \mathrm{d} \sigma)$, see [Nec67, Chap. $2 \S 6$, Theorem 6.2].

Let $\mathcal{A}(t)$ be the operator associated with $\mathfrak{a}(t, .,$.$) and \mathcal{B}(t)$ the operator associated with $\mathfrak{b}(t, .,$.$) . Note that the part A(t)$ in $H:=L^{2}(\Omega)$ of $\mathcal{A}(t)$ is interpreted as (minus) the Laplacian with time dependent Robin boundary conditions

$$
\partial_{\nu} v+\beta_{1}(t, .) v=0 \text { on } \Gamma .
$$


Here we use the following weak definition of the normal derivative. Let $v \in$ $H^{1}(\Omega)$ such that $\Delta v \in L^{2}(\Omega)$. Let $h \in L^{2}(\Gamma, \mathrm{d} \sigma)$. Then $\partial_{\nu} v=h$ by definition if $\int_{\Omega} \nabla v \nabla w+\int_{\Omega} \Delta v w=\int_{\Gamma} h w \mathrm{~d} \sigma$ for all $w \in H^{1}(\Omega)$. Based on this definition, the domain of $A(t)$ is the set

$$
D(A(t))=\left\{v \in H^{1}(\Omega): \Delta v \in L^{2}(\Omega), \partial_{\nu} v+\left.\beta_{1}(t) v\right|_{\Gamma}=0\right\},
$$

and for $v \in D(A(t))$ the operator is given by $A(t) v=-\Delta v$.

Maximal regularity on $H$ for the first order Cauchy problem associated with $A(t)$ was proved in [ADLO13]. Here we study the second order problem. By Theorem 4.1, the damped wave equation

$$
\left\{\begin{array}{l}
\ddot{u}(t)-\Delta \dot{u}(t)-\Delta u(t)=f(t) \\
u(0)=u_{0}, \quad \dot{u}(0)=u_{1} \in H^{1}(\Omega) \\
\partial_{\nu}(\dot{u}(t)+u(t))+\beta_{2}(t, .) \dot{u}(t)+\beta_{1}(t, .) u(t)=0 \text { on } \Gamma
\end{array}\right.
$$

has a unique solution $u \in M R(V, V, H)=H^{2}\left(0, T ; L^{2}(\Omega)\right) \cap H^{1}\left(0, T ; H^{1}(\Omega)\right)$ whenever $f \in L^{2}\left(0, T, L^{2}(\Omega)\right)$.

Indeed, Theorem 4.1 implies that there exists $u \in M R\left(V, V, V^{\prime}\right)$ with $u(0)=$ $u_{0}, \dot{u}(0)=u_{1}$ and

$$
(\ddot{u}, v)_{H}+\mathfrak{b}(t, \dot{u}, v)+\mathfrak{a}(t, u, v)=(f, v)_{H}
$$

for all $v \in V$ and all $t \in[0, T] \backslash N$, where $N$ is a Lebesgue null set. Let $t \in[0, T] \backslash N$, then for the special choice $v \in \mathcal{D}(\Omega)$ we obtain that (6.5) implies $\ddot{u}(t)-\Delta \dot{u}(t)-\Delta u(t)=f(t)$. This together with (6.5) and the above definition of the normal derivative shows

$$
\partial_{\nu}(\dot{u}(t)+u(t))+\beta_{2}(t, .) \dot{u}(t)+\beta_{1}(t, .) u(t)=0 \text { on } \Gamma .
$$

II) A quasi-linear problem.

Let $\Omega$ be a bounded open set of $\mathbb{R}^{d}$ and let $H$ be the real-valued Hilbert space $L^{2}(\Omega, \mathrm{d} x)$ and $V$ be a closed subspace of $H^{1}(\Omega)$ which contains $H_{0}^{1}(\Omega)$. If $V \neq$ $H_{0}^{1}(\Omega)$ we assume that $\Omega$ is a Lipschitz domain to ensure that the embedding of $V$ in $H$ is compact. This latter property is always true for $V=H_{0}^{1}(\Omega)$ for any bounded domain $\Omega$.

For $g, h \in L^{2}(0, T ; H)$ we define the forms $\mathfrak{a}_{g, h}, \mathfrak{b}_{g, h}:[0, T] \times V \times V \rightarrow \mathbb{R}$ by

$$
\mathfrak{a}_{g, h}(t, u, v)=\sum_{k, j=1}^{d} \int_{\Omega} a_{j k}(t, x, g, h) \partial_{k} u \partial_{j} v \mathrm{~d} x
$$

and

$$
\mathfrak{b}_{g, h}(t, u, v)=\sum_{k, j=1}^{d} \int_{\Omega} b_{j k}(t, x, g, h) \partial_{k} u \partial_{j} v \mathrm{~d} x .
$$

We assume that the coefficients $a_{j k}, b_{j k}:[0, T] \times \Omega \times \mathbb{R} \times \mathbb{R} \rightarrow \mathbb{R}$ are uniformly bounded on $[0, T] \times \Omega \times \mathbb{R} \times \mathbb{R}$ by a constant $M>0$ and satisfy the usual ellipticity condition

$$
\sum_{k, j=1}^{d} a_{j k}(t, x, y, z) \xi_{k} \xi_{j} \geq \eta|\xi|^{2}, \quad \sum_{k, j=1}^{d} b_{j k}(t, x, y, z) \xi_{k} \xi_{j} \geq \eta|\xi|^{2}
$$


for a.e. $(t, x) \in[0, T] \times \Omega$ and all $y, z \in \mathbb{R}, \xi \in \mathbb{R}^{d}$. Here $\eta>0$ is a constant. Moreover we assume that $a_{j k}(t, x, .,),. b_{j k}(t, x, . .$.$) are continuous for a.e. (t, x)$. We denote by $\mathcal{A}_{g, h}(t)$ and $\mathcal{B}_{g, h}(t)$ the associated operators.

Given $u_{0} \in V, u_{1} \in H$ and $f \in L^{2}\left(0, T ; V^{\prime}\right)$ the second order Cauchy problem

$$
\left\{\begin{array}{l}
\ddot{u}(t)+\mathcal{B}_{g, h}(t) \dot{u}(t)+\mathcal{A}_{g, h}(t) u(t)=f(t) \quad t \text {-a.e. } \\
u(0)=u_{0}, \dot{u}(0)=u_{1}
\end{array}\right.
$$

has a unique solution $u_{g, h} \in M R\left(V, V, V^{\prime}\right)$ by Theorem 3.1. Moreover, by Proposition 3.3 there exists $C>0$ and $0<T_{0} \leq T$ depending only on $M$ and $\eta$ such that the solution of $(6.6)$ on $\left[0, T_{0}\right]$ satisfies the estimate

$$
\left\|u_{g, h}\right\|_{M R_{T_{0}}\left(V, V, V^{\prime}\right)} \leq C\left[\left\|u_{0}\right\|_{V}+\left\|u_{1}\right\|_{H}+\|f\|_{L^{2}\left(0, T_{0} ; V^{\prime}\right)}\right] .
$$

Note that $C$ and $T_{0}$ are independent of $g$ and $h$. We want to show that the quasi-linear problem

$$
\left\{\begin{array}{l}
\ddot{u}(t)+\mathcal{B}_{u, \dot{u}}(t) \dot{u}(t)+\mathcal{A}_{u, \dot{u}}(t) u(t)=f(t) \quad t \text {-a.e. } \\
u(0)=u_{0}, \dot{u}(0)=u_{1}
\end{array}\right.
$$

has a solution $u$ in $M R\left(V, V, V^{\prime}\right)$. We define the mapping $S: H^{1}(0, T ; H) \rightarrow$ $H^{1}(0, T ; H)$ by $S g:=u_{g, \dot{g}}$. Note that by (6.7) and the fact that $C$ is independent of $g$ and $h, \operatorname{Im}(S)$ is a bounded subset of $M R\left(V, V, V^{\prime}\right)$. Moreover by AubinLions lemma, $M R\left(V, V, V^{\prime}\right)$ is compactly embedded into $H^{1}(0, T ; H)$. Therefore, if $S$ is continuous then we can apply Schauder's fixed point theorem to obtain $u \in H^{1}(0, T ; H)$ such that $S u=u$. Thus $u$ is also in $M R\left(V, V, V^{\prime}\right)$ and $u \in$ $\operatorname{Im}(S)$. Hence $u$ is a solution of (6.8).

It remains to prove that $S$ is continuous. Let $g_{n} \rightarrow g$ in $H^{1}(0, T ; H)$ and set $u_{n}:=S g_{n}$. Since a sequence converges to a fixed element $u$ if and only if each subsequence has a subsequence converging to $u$ we may deliberately take subsequences. Since $L^{2}(0, T ; H)$ is isomorphic to $L^{2}((0, T) \times \Omega)$ we may assume (after taking a sub-sequence) that $g_{n} \rightarrow g$ and $\dot{g}_{n} \rightarrow \dot{g}$ for a.e. $(t, x)$. Furthermore since the sequence $u_{n}$ is bounded in $M R\left(V, V, V^{\prime}\right)$ we may assume (after taking a sub-sequence) that $u_{n} \rightarrow u$ in $H^{1}(0, T ; H)$ and $u_{n} \rightarrow u$ in $M R\left(V, V, V^{\prime}\right)$. Hence $a_{j k}\left(t, x, g_{n}, \dot{g}_{n}\right) \rightarrow a_{j k}(t, x, g, \dot{g})$ and $b_{j k}\left(t, x, g_{n}, \dot{g}_{n}\right) \rightarrow$ $b_{j k}(t, x, g, \dot{g})$ for a.e. $(t, x)$. Now the equality $u_{n}=S g_{n}$ means that

$$
\begin{aligned}
& \left\langle\ddot{u}_{n}, v\right\rangle_{L^{2}\left(0, T ; V^{\prime}\right), L^{2}(0, T ; V)}+\sum_{j, k=1}^{d}\left(\partial_{j} \dot{u}_{n} \mid b_{j k}\left(t, x, g_{n}, \dot{g}_{n}\right) \partial_{k} v\right)_{L^{2}(0, T ; H)} \\
& \quad+\sum_{j, k=1}^{d}\left(\partial_{j} u_{n} \mid a_{j k}\left(t, x, g_{n}, \dot{g}_{n}\right) \partial_{k} v\right)_{L^{2}(0, T ; H)}=\langle f, v\rangle_{L^{2}\left(0, T ; V^{\prime}\right), L^{2}(0, T ; V)}
\end{aligned}
$$

for all $v \in L^{2}(0, T ; V)$ and $u_{n}(0)=u_{0}, \dot{u}_{n}(0)=u_{1}$. By the dominated convergence theorem $a_{j k}\left(t, x, g_{n}, \dot{g}_{n}\right) \partial_{k} v \rightarrow a_{j k}(t, x, g, \dot{g}) \partial_{k} v$ in $L^{2}(0, T ; H)$. Moreover $u_{n} \rightarrow u$ in $M R\left(V, V, V^{\prime}\right)$ implies that $\partial_{j} u_{n} \rightarrow \partial_{j} u$ and $\partial_{j} \dot{u}_{n} \rightarrow \partial_{j} \dot{u}$ in $L^{2}(0, T ; H)$. Thus taking the limit for $n \rightarrow \infty$ yields

$$
\langle\ddot{u}, v\rangle_{L^{2}\left(0, T ; V^{\prime}\right), L^{2}(0, T ; V)}+\sum_{j, k=1}^{d}\left(\partial_{j} \dot{u} \mid b_{j k}(t, x, g, \dot{g}) \partial_{k} v\right)_{L^{2}(0, T ; H)}
$$




$$
+\sum_{j, k=1}^{d}\left(\partial_{j} u \mid a_{j k}(t, x, g, \dot{g}) \partial_{k} v\right)_{L^{2}(0, T ; H)}=\langle f, v\rangle_{L^{2}\left(0, T ; V^{\prime}\right), L^{2}(0, T ; V)}
$$

for all $v \in L^{2}(0, T ; V)$ and $u(0)=u_{0}, \dot{u}(0)=u_{1}$. Note that for the initial condition we have used that $M R\left(V, V, V^{\prime}\right) \hookrightarrow C^{1}([0, T] ; H) \cap C([0, T] ; V)$, see (3.3). This is equivalent to $S g=u$. Hence $S$ is continuous.

\section{References}

[ADLO13] W. Arendt, D. Dier, H. Laasri and E.M. Ouhabaz. Maximal regularity for evolution equations governed by non-autonomous forms. Preprint 2013, available at http://arxiv.org/abs/1303.1166.

[BCS08] C.J.K. Batty, R. Chill and S. Srivastava. Maximal regularity for second order non-autonomous Cauchy problems. Studia Math. 189 (2008) no 3, 205-223.

[DL88] R. Dautray and J.L. Lions. Analyse Mathématique et Calcul Numérique pour les Sciences et les Techniques. Vol. 8, Masson, Paris, 1988.

[DL92] R. Dautray and J.L. Lions. Mathematical Analysis and Numerical Methods for Science and Technology. Vol. 5. Evolution problems I, SpringerVerlag, Berlin, 1992.

[Lio59] J.L. Lions. Problemi misti nel senso di Hadamard classici e generalizzati. Milan Journal of Mathematics 28 (1958), 147-188.

[Lio61] J.L. Lions. Equations Différentielles Opérationnelles et Problèmes aux Limites. Springer-Verlag, Berlin, Göttingen, Heidelberg, 1961.

[Nec67] J. Necaš. Les Méthodes Directes en Théorie des Equations Elliptiques. Masson Paris 1967.

[OS10] E. M. Ouhabaz and C. Spina. Maximal regularity for nonautonomous Schrödinger type equations. J. Differential Equations 248 (2010),16681683.

[Paz83] A. Pazy. Semigroups of Linear Operators and Applications to Partial Differential Equations. Springer-Verlag, Berlin, 1983.

[Sho97] R. E. Showalter. Monotone Operators in Banach Space and Nonlinear Partial Differential Equations. Mathematical Surveys and Monographs. American Mathematical Society, Providence, RI, 1997.

Dominik Dier, Institute of Applied Analysis, University of Ulm, 89069 Ulm, Germany,

dominik.dier@uni-ulm.de

El Maati Ouhabaz, Institut de Mathématiques (IMB), Univ. Bordeaux, 351, cours de la Libération, 33405 Talence cedex, France,

Elmaati.Ouhabaz@math.u-bordeaux1.fr 